\title{
Application of Effect-Compartment Model to Bumetanide-Indomethacin Interaction in Dogs
}

\author{
David E. Smith, ${ }^{1,2}$ Henry S. H. Lau, ${ }^{1}$ and Jeffrey L. Fox ${ }^{1,3}$ \\ Received November 17, 1982-Final May 27, 1983
}

\begin{abstract}
Analyses of bumetanide's dose-response relationship have been complicated by the hysteresis observed between the drug's urinary excretion rate and its sodium excretion. This apparent time lag reflects the disequilibrium between the urine concentration and effect compartment (biophase) which occurs during the early distribution phase. In the present article, an expanded pharmacodynamic model has been introduced in which the hypothetical effect compartment is linked, by a first-order process $\left(\mathrm{K}_{\mathrm{ue}}\right)$, to the urine compartment. Drug dissipation from the effect compartment occurs by means of the first-order rate constant, $\mathrm{K}_{\mathrm{eo}}$. This representation accommodates bumetanide's luminal site of action in the kidney tubule as well as the drug's temporal component. Application of this model to the bumetanide-indomethacin interaction in dogs is examined.
\end{abstract}

KEY WORDS: bumetanide; indomethacin; effect-compartment model; dose-response relationship.

\section{INTRODUCTION}

Bumetanide is a new diuretic which inhibits solute reabsorption in the thick ascending limb of the loop of Henle and to a lesser extent in the proximal tubule (1-4). There, the drug exerts its natriuretic and diuretic effect from the luminal surface of the kidney nephron (3). In addition, indirect studies with indomethacin have suggested that the pharmacodynamic response to bumetanide may be prostaglandin-mediated (5-9).

\footnotetext{
This work was supported in part by a Biomedical Research Support Grant from the College of Pharmacy and a Faculty Research Rackham Grant, The University of Michigan. During the course of this work, H. S. H. Lau received support as a National Institutes of Health Predoctoral Scholar on NIH Training Grant GM 07767-04.

${ }^{1}$ College of Pharmacy, The University of Michigan, Ann Arbor, Michigan 48109.

${ }^{2}$ Author to whom correspondence should be addressed.

${ }^{3}$ Present address: Department of Pharmaceutics, College of Pharmacy, The University of Utah, Salt Lake City, Utah 84112.
} 
Previous studies $(5-8,10-13)$ have been vague and conflicting in attempting to define the determinants of bumetanide response. This is not surprising since these investigators did not measure the concentrations of bumetanide in a sampling compartment reflective of the drug's site of action. Nevertheless, we have subsequently demonstrated (a) that bumetanide urinary excretion rate was a better indicator of natriuresis than was plasma concentration (14), and (b) that indomethacin diminishes the pharmacodynamic response to bumetanide by a mechanism other than prostaglandinmediated changes in renal hemodynamics (9). However, in the one study (14) we commented that data analyses were complicated by the hysteresis or time lag observed between the urinary excretion rate of bumetanide and sodium excretion during the early time periods. A similar hysteresis was also observed when bumetanide was administered concomitantly with indomethacin (9) and reflects the disequilibrium between the urine concentration and effect compartment (biophase) which occurs during the early distribution phase.

In the present article, we have expanded upon a previously proposed pharmacodynamic model by Sheiner and coworkers (15-17). However, the hypothetical effect compartment is now connected to the urine compartment, as opposed to the plasma compartment. The revised model which we propose should be valuable in elucidating the sensitivity as well as the temporal components of bumetanide response. More importantly, the proposed model affords a new method for interpreting the data of luminally active drugs which exhibit a hysteresis between the urine and effect compartments. The application of this model to the bumetanide-indomethacin interaction in dogs is examined.

\section{EXPERIMENTAL PROCEDURE}

\section{Materials and Methods}

Details of the experimental protocol, drug administration schedules, and analytical methodologies have been previously reported (9). We summarize briefly. Four male, mongrel, conditioned, unanesthetized dogs weighing $22.0-29.0 \mathrm{~kg}$ received $0.250 \mathrm{mg} / \mathrm{kg}$ of intravenous bumetanide before (treatment I) and after (treatment II) indomethacin pretreatment. Lactated Ringer's solution was administered by vein throughout the entire study period for both treatments I and II at a flow rate of $2 \mathrm{ml} / \mathrm{min}$ to avoid fluid and electrolyte depletion. Serial plasma and urine samples containing bumetanide as well as indomethacin plasma concentrations were 
determined using a high-performance liquid chromatographic (HPLC) technique. Urinary sodium was measured by flame photometry.

\section{Pharmacodynamic Model}

Sheiner and coworkers (15-17) have recently proposed a hypothetical effect-compartment model which characterizes the temporal aspects of pharmacodynamics as well as the time-independent sensitivity component. The effect compartment is modeled as an additional compartment which is linked to the central compartment by a first-order process. However, the exponential term associated with the effect compartment does not enter the pharmacokinetic solution since the loss of actual drug mass from the central compartment is negligible. In the present model (Fig. 1), we have expanded this concept by linking the hypothetical effect compartment to the urine compartment with the first-order rate constant, $K_{u e}$. Drug dissipation from the effect compartment occurs by means of the first-order rate constant, $K_{e o}$, which allows one to precisely characterize the temporal aspects of equilibrium between urinary excretion rate and effect.

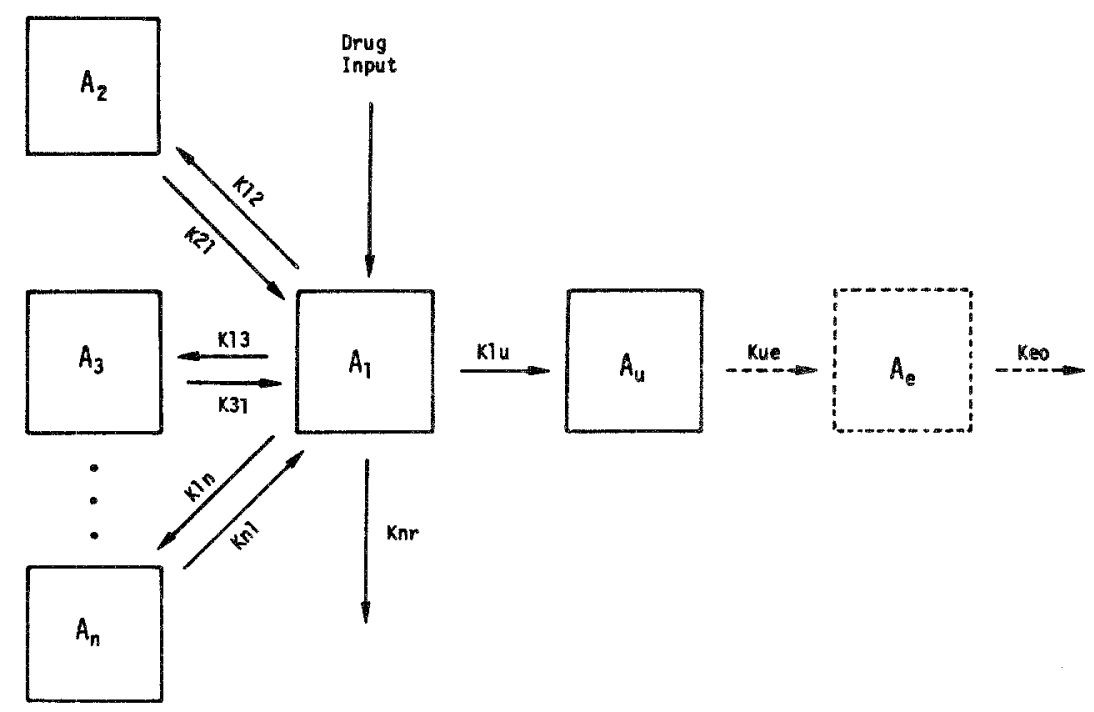

Fig. 1. Proposed pharmacodynamic model, viewed as an $n$-compartment mammillary representation, where the effect compartment is linked by a first-order process to the urine compartment. See Appendix for explanation of symbols. 
Equations (1) and (2) describe the change in amount of drug in the effect compartment with time for the intravenous infusion of a drug into the central compartment when fitted to a bi- and triexponential pharmacokinetic model, respectively: ${ }^{4}$

$$
\begin{aligned}
& \frac{d A_{e}}{d t}=K_{u e} K_{1 u} K^{0}\left[\frac{\left(e^{b \lambda_{1}}-1\right)\left(K_{21}-\lambda_{1}\right) e^{--\lambda_{1} t}}{\lambda_{1}\left(\lambda_{2}-\lambda_{1}\right)\left(K_{e o}-\lambda_{1}\right)}+\frac{\left(e^{b \lambda_{2}}-1\right)\left(K_{21}-\lambda_{2}\right) e^{-\lambda_{2} t}}{\lambda_{2}\left(\lambda_{1}-\lambda_{2}\right)\left(K_{e o}-\lambda_{2}\right)}\right. \\
& \left.+\frac{\left(e^{b K_{e o}}-1\right)\left(K_{21}-K_{e o}\right) e^{-K_{e o} t}}{K_{e o}\left(\lambda_{1}-K_{e o}\right)\left(\lambda_{2}-K_{e o}\right)}\right] \\
& \frac{d A_{e}}{d t}=K_{u e} K_{1 u} K^{0}\left[\frac{\left(e^{b \lambda_{1}}-1\right)\left(K_{21}-\lambda_{1}\right)\left(K_{31}-\lambda_{1}\right) e^{-\lambda_{1} t}}{\lambda_{1}\left(\lambda_{2}-\lambda_{1}\right)\left(\lambda_{3}-\lambda_{1}\right)\left(K_{e o}-\lambda_{1}\right)}\right. \\
& +\frac{\left(e^{b \lambda_{2}}-1\right)\left(K_{21}-\lambda_{2}\right)\left(K_{31}-\lambda_{2}\right) e^{-\lambda_{2} t}}{\lambda_{2}\left(\lambda_{1}-\lambda_{2}\right)\left(\lambda_{3}-\lambda_{2}\right)\left(K_{e o}-\lambda_{2}\right)} \\
& +\frac{\left(e^{b \lambda_{3}}-1\right)\left(K_{21}-\lambda_{3}\right)\left(K_{31}-\lambda_{3}\right) e^{-\lambda_{3} t}}{\lambda_{3}\left(\lambda_{1}-\lambda_{3}\right)\left(\lambda_{2}-\lambda_{3}\right)\left(K_{e o}-\lambda_{3}\right)} \\
& \left.+\frac{\left(e^{b K_{e o}}-1\right)\left(K_{21}-K_{e o}\right)\left(K_{31}-K_{e o}\right) e^{-K_{e o} t}}{K_{e o}\left(\lambda_{1}-K_{e o}\right)\left(\lambda_{2}-K_{e o}\right)\left(\lambda_{3}-K_{e o}\right)}\right]
\end{aligned}
$$

In Eqs. (1) and (2), there are two unknown parameters, $K_{u e}$ and $K_{e o}$. All other pharmacokinetic parameters were previously introduced (9). $K_{u e}$ was arbitrarily assigned a value of $0.001 \mathrm{~min}^{-1}$ in the subsequent analyses for two reasons. First, since the transfer of mass to the effect compartment is negligible, the rate constant connecting the urine compartment to the effect compartment is assumed to be very small relative to the magnitude of the smallest rate constant of the pharmacokinetic model. Hence, under this assumption, the exact magnitude of $K_{u e}$ is inconsequential (15). Second, since we are treating the data on a comparative basis, the exact value of $K_{u e}$ is not important as long as it is consistent between treatments I and II.

Substitution of $d A_{e} / d t$ from Eqs. (1) and (2), depending upon the pharmacokinetic model utilized, was then made into the sigmoid $E_{\max }$ model, as shown below (see Appendix for explanation of symbols):

$$
E=\frac{E_{\max }}{\left[\left(\frac{A_{e 50}}{d A_{e} / d t}\right)^{\gamma}+1\right]}+E_{0}
$$

Equation (3) defines the relationship between the observed pharmacological effect $(E)$ and the predicted rate of change of drug in the effect compartment $\left(d A_{e} / d t\right)$. Using the sodium excretion rate (observed effect) of bumetanide with time as input, the unknown parameters $\left(K_{e o}, E_{\max }, A_{e 50}, E_{0}, \gamma\right)$ were ${ }^{4}$ See Appendix for the derivation of Eqs. (1) and (2) and for an explanation of symbols. 
estimated for each dog by a nonlinear least-squares regression program (J. L. Fox) using the Amdahl 470v/6 digital computer. The regression program employs a Levenberg-Marquardt minimization technique and is essentially an adaptation of an algorithm for systems of nonlinear equations $(18,19)$ to the present case, where the system is overdetermined and a sum of squares minimization is sought. This algorithm combines the rapid convergence properties of a Gauss-Newton procedure when the solution is near, with the slow but more likely convergence of a steepest descent procedure when far from the solution. A special matrix updating procedure (20) permits the Jacobian matrix to be economically updated on each iteration with no additional function evaluations required. Statistical analyses of the calculations were identical with those produced by Metzler's program (21).

Six data sets were fitted to a pharmacodynamic model incorporating a biexponential pharmacokinetic equation, and two data sets were fitted to a model incorporating a triexponential pharmacokinetic equation. All pharmacodynamic and previously fitted pharmacokinetic (9) models utilized a weighting factor of unity. Other pharmacodynamic models were tested ( $E_{\max }$ and $\log$-linear models), but the data did not fit them as well, as determined by the values for $R^{2}$ and CORR. Furthermore, Eq. (3) allows for a sigmoidal shape of the response curve that is not allowed by the simple $E_{\max }$ model, and it is applicable outside the $20-80 \%$ effect range to which the log-linear method is limited.

\section{RESULTS}

The pharmacodynamic parameter estimates are displayed in Table I. There was no significant difference (as determined by a paired $t$ test) in $K_{e o}$ between treatments I and II, and the mean equilibrium half-lives $\left(0.693 / K_{\text {eo }}\right)$ were 36.5 and $41.8 \mathrm{~min}$, respectively. In addition, the confidence in the accuracy of $K_{e o}$ is quite high since the intraanimal coefficients of variation $(C V \mathrm{~s})$ for this parameter were less than $7 \%$. On the other hand, little confidence can be placed in the values of the other estimated parameters $\left(E_{\max }, A_{e 50}, E_{0}, \gamma\right)$ due to the large variability surrounding them ( $C V \mathrm{~s}$ greater than $100 \%$ in many cases). This probably reflects the uncertainty in estimating five unknown parameters in each animal with only six data points, as well as the uncertainty in characterizing the maximal response to drug in the effect compartment. Despite this handicap, these parameters were useful in the interpolation, as opposed to extrapolation, of the predicted pharmacodynamic effect. Figure 2 displays the ability of the model to accurately predict, as determined by the coefficient of determination, the sodium excretion rate of bumetanide using pooled data from both treatments $\left(r^{2}=0.933 ; p<0.001\right)$. 


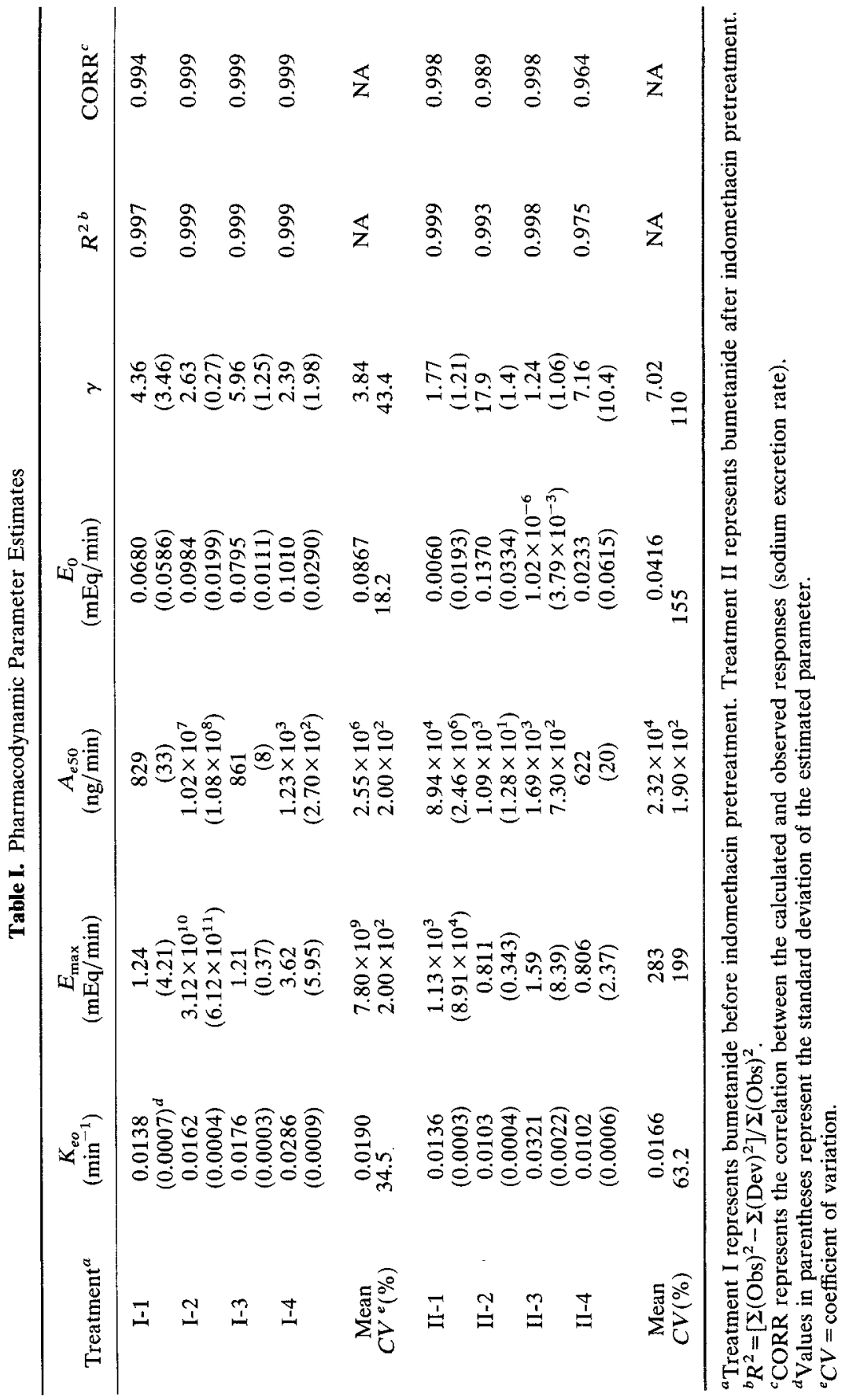




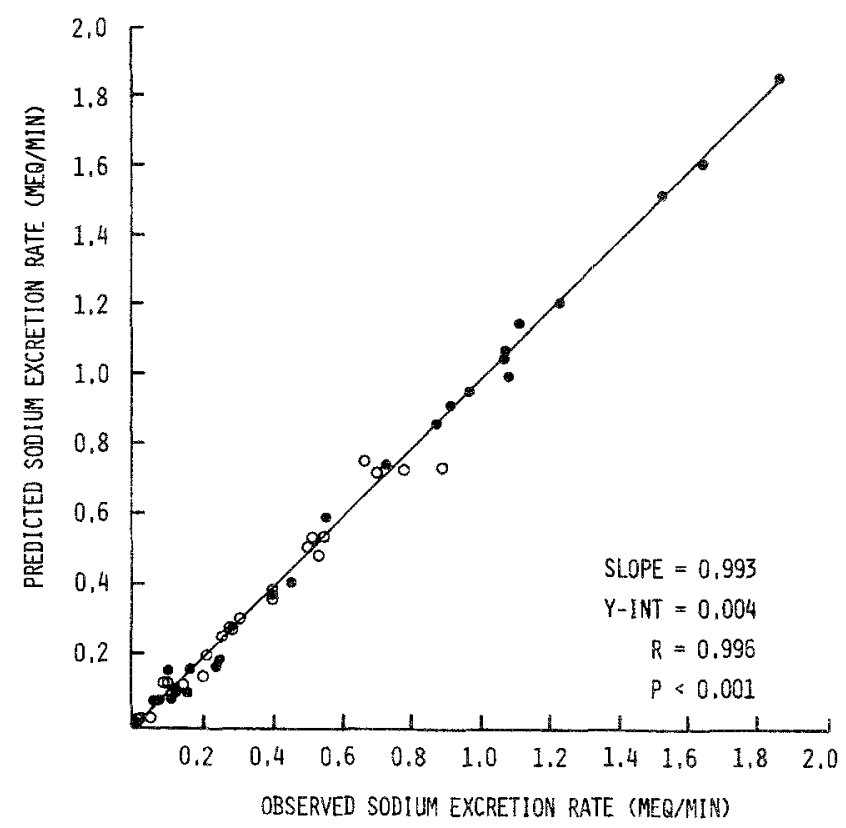

Fig. 2. Predicted vs observed pharmacodynamic effect (sodium excretion rate) using pooled data of bumetanide alone ( ) and bumetanide after indomethacin pretreatment $(O)$. The predicted effect was determined with Eq. (3).

Figure 3 demonstrates that a time lag exists between the bumetanide urinary excretion rate and sodium excretion during the early time periods (first $40 \mathrm{~min}$ ). Although the excretion rate of bumetanide is greatest at $10 \mathrm{~min}$, the peak effect occurs approximately $30 \mathrm{~min}$ after drug dosing. However, after about $40 \mathrm{~min}$ the curve for sodium excretion rate begins to parallel that of bumetanide excretion rate. This time lag was evident during indomethacin pretreatment (9) as well as during the concomitant administration of bumetanide and probenecid (14).

Figure 4 demonstrates that the time course of $d A_{e} / d t$, in contrast to bumetanide urinary excretion rate, follows a pattern similar to that of sodium excretion rate over the entire dose-response curve. This finding supports the premise that bumetanide hysteresis is a consequence of disequilibrium between drug in the urine and effect compartments during bumetanide's early distribution phase. It is also observed that the rate of change of drug in the effect compartment does not differ between treatments $I$ and II. Nevertheless, indomethacin pretreatment resulted in a dramatic reduction of bumetanide-induced sodium excretion $(121 \pm 17$ for treatment I vs $62.4 \pm 21.3 \mathrm{mEq} / 4 \mathrm{hr}$ for treatment II; $p<0.005$; ref. 9). The effect 


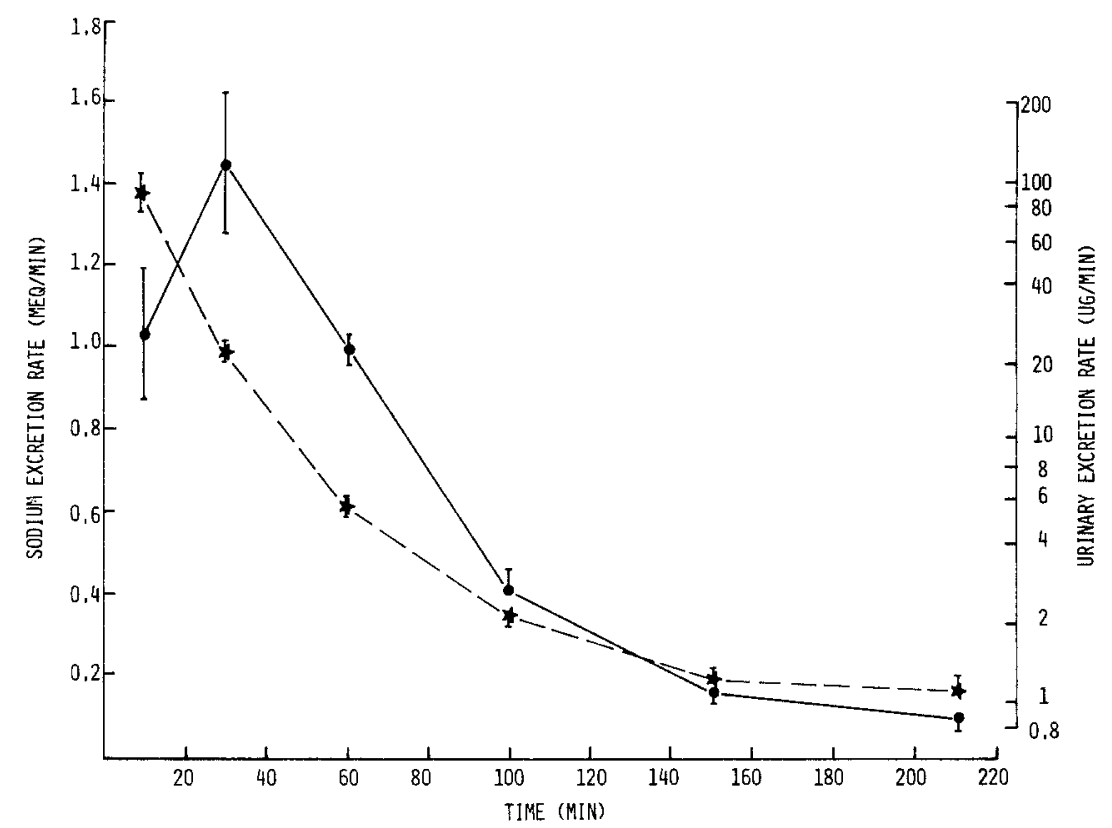

Fig. 3. Sodium excretion rate vs midpoint time plot (-) and urinary excretion rate vs midpoint time plot $(\star-\cdots \star)$ of bumetanide alone. Data are expressed as the mean $\pm \operatorname{SEM}(n=4)$.

of indomethacin on the dose-response curves of bumetanide is further illustrated in Fig. 5. Bumetanide response, in the presence of indomethacin, was significantly reduced during the first 40-60 min following bumetanide dosing (two highest values for $d A_{e} / d t$ ), even though the rates of change of bumetanide in the effect compartment were equivalent between treatments.

\section{DISCUSSION}

The determinants of bumetanide response have been poorly characterized in previous studies $(5-8,10-13)$ due to the inability of these investigators to measure bumetanide concentrations in the plasma and urine. Furthermore. standard pharmacodynamic models (22) do not take into account any lag of effect behind changes in the plasma or urine concentrations during periods of disequilibrium. Therefore, an expanded pharmacodynamic model has been introduced in which the hypothetical effect compartment is linked, by a first-order process, to the urine compartment. This representation accommodates bumetanide's luminal site of action in the kidney tubule as well as the drug's temporal component. 


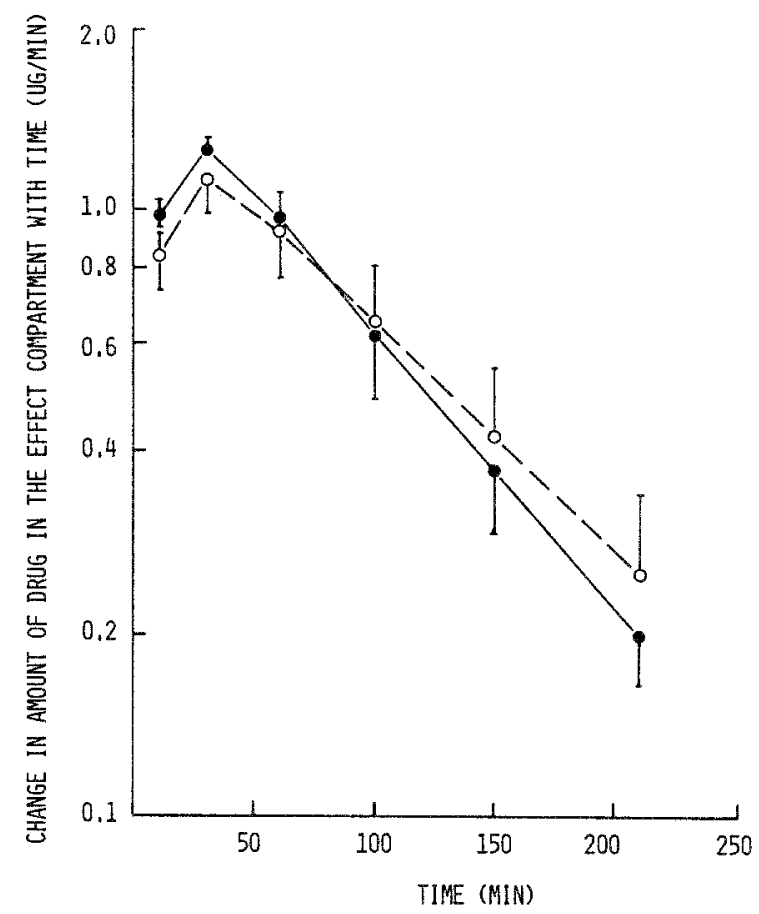

Fig. 4. Predicted rate of change of drug in the effect compartment vs midpoint time plots of bumetanide alone (-) and bumetanide after indomethacin pretreatment $(0-.0)$. Data are expressed as the mean \pm SEM $(n=4)$.

Two mechanisms are consistent with the attenuation of bumetanide's natriuretic and diuretic effects by indomethacin. These include the inhibition of prostaglandin synthesis by indomethacin (pharmacodynamic interaction) as well as a possible competition between bumetanide and indomethacin for active tubular secretion into the kidney lumen (pharmacokinetic interaction). The present analyses corroborate with those of our previous study (9) and further support the tenet that indomethacin diminishes the pharmacodynamic response to bumetanide via the inhibition of prostaglandin synthesis.

The proposed pharmacodynamic model also clarifies the mechanism for the apparent time lag between bumetanide's effect and the drug's concentration. Even though the absolute values of the pharmacodynamic parameters $\left(E_{\max }, A_{e 50}, E_{0}, \gamma\right)$ were not well characterized for reasons previously discussed, the most important parameter, $K_{e o}$, was very well characterized. The ability to determine this parameter alone is significant 


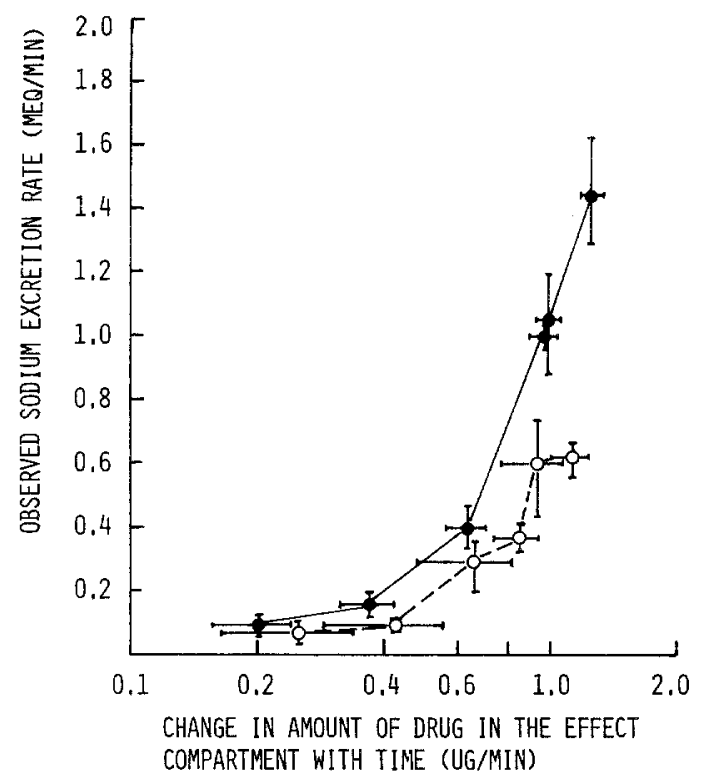

Fig. 5. Observed sodium excretion rate vs predicted rate of change of drug in the effect compartment plots of bumetanide alone (-) and bumetanide after indomethacin pretreatment $(\mathrm{O}--\mathrm{O})$. Data are expressed as the mean $\pm \operatorname{SEM}(n=4)$.

for several reasons. First, it quantifies the equilibrium half-life between drug in the urine (for the proposed model) and drug in the biophase. Second, it helps to clarify some of the discrepancies observed in attempting to elucidate the determinants of bumetanide's response. And third, this disequilibrium concept is of import when designing future experiments in which bumetanide effect is being correlated with the drug's urinary excretion.

In general, the diuretic must reside in the body for a minimum of 3.32 equilibration half-lives in order to achieve $90 \%$ equilibration between the urine and effect compartments. Only when distribution equilibrium has occurred will the urinary excretion rate of bumetanide correlate with the drug's effect at all times (assuming no pharmacodynamic interaction). We are currently performing steady-state experiments so that the dose-response relationship of bumetanide can be defined in the absence of complicating kinetic factors. Furthermore, and most importantly, the proposed pharmacodynamic model introduces a novel method for interpreting the data of any luminally active drug which exhibits a hysteresis similar to that of bumetanide. 


\section{APPENDIX}

\section{Solving the Pharmacodynamic Model (Figure 1)}

The Laplace transform for the amount of drug in the effect compartment is given by the product of the input and disposition functions:

$$
a_{s, e}=(i n s)(d s, e)
$$

The input function for an intravenous infusion which begins at time zero is

$$
\text { ins }=K^{0}\left(1-e^{-b s}\right) / s
$$

The disposition function for the effect compartment is

$$
d s, e=\frac{K_{u e}(d s, u)}{\left(s+K_{e o}\right)}
$$

where

$$
d s, u=\frac{K_{1 u}(d s, 1)}{s}
$$

and

$$
d s, 1=\frac{\left(s+K_{21}\right)\left(s+K_{31}\right)}{\left(s+\lambda_{1}\right)\left(s+\lambda_{2}\right)\left(s+\lambda_{3}\right)}
$$

Substitution of Eqs. (5)-(8) into Eq. (4) results in Eq. (9):

$$
a_{s, e}=\frac{K_{u e} K_{1 u} K^{0}\left(1-e^{-b s}\right)\left(s+K_{21}\right)\left(s+K_{31}\right)}{s^{2}\left(s+\lambda_{1}\right)\left(s+\lambda_{2}\right)\left(s+\lambda_{3}\right)\left(s+K_{e o}\right)}
$$

The anti-Laplace of Eq. (9) is

$$
\begin{aligned}
A_{e}= & K_{u e} K_{1 u} K^{0}\left[\frac{b K_{21} K_{31}}{\lambda_{1} \lambda_{2} \lambda_{3} K_{e o}}+\frac{\left(1-e^{b \lambda_{1}}\right)\left(K_{21}-\lambda_{1}\right)\left(K_{31}-\lambda_{1}\right) e^{-\lambda_{1} t}}{\lambda_{1}^{2}\left(\lambda_{2}-\lambda_{1}\right)\left(\lambda_{3}-\lambda_{1}\right)\left(K_{e o}-\lambda_{1}\right)}\right. \\
& +\frac{\left(1-e^{b \lambda_{2}}\right)\left(K_{21}-\lambda_{2}\right)\left(K_{31}-\lambda_{2}\right) e^{-\lambda_{2} t}}{\lambda_{2}^{2}\left(\lambda_{1}-\lambda_{2}\right)\left(\lambda_{3}-\lambda_{2}\right)\left(K_{e o}-\lambda_{2}\right)} \\
& +\frac{\left(1-e^{b \lambda_{3}}\right)\left(K_{21}-\lambda_{3}\right)\left(K_{31}-\lambda_{3}\right) e^{-\lambda_{3} t}}{\lambda_{3}^{2}\left(\lambda_{1}-\lambda_{3}\right)\left(\lambda_{2}-\lambda_{3}\right)\left(K_{e o}-\lambda_{3}\right)} \\
& \left.+\frac{\left(1-e^{b K_{e o}}\right)\left(K_{21}-K_{e o}\right)\left(K_{31}-K_{e o}\right) e^{-K_{e o} t}}{K_{e o}^{2}\left(\lambda_{1}-K_{e o}\right)\left(\lambda_{2}-K_{e o}\right)\left(\lambda_{3}-K_{e o}\right)}\right]
\end{aligned}
$$


The derivative of Eq. (10) with respect to time results in the following:

$$
\begin{aligned}
& \frac{d A_{e}}{d t}=K_{u e} K_{1 u} K^{0}\left[\frac{\left(e^{b \lambda_{1}}-1\right)\left(K_{21}-\lambda_{1}\right)\left(K_{31}-\lambda_{1}\right) e^{-\lambda_{1} t}}{\lambda_{1}\left(\lambda_{2}-\lambda_{1}\right)\left(\lambda_{3}-\lambda_{1}\right)\left(K_{e o}-\lambda_{1}\right)}\right. \\
& +\frac{\left(e^{b \lambda_{2}}-1\right)\left(K_{21}-\lambda_{2}\right)\left(K_{31}-\lambda_{2}\right) e^{-\lambda_{2} t}}{\lambda_{2}\left(\lambda_{1}-\lambda_{2}\right)\left(\lambda_{3}-\lambda_{2}\right)\left(K_{e o}-\lambda_{2}\right)} \\
& +\frac{\left(e^{b \lambda_{3}}-1\right)\left(K_{21}-\lambda_{3}\right)\left(K_{31}-\lambda_{3}\right) e^{-\lambda_{3} t}}{\lambda_{3}\left(\lambda_{1}-\lambda_{3}\right)\left(\lambda_{2}-\lambda_{3}\right)\left(K_{e o}-\lambda_{3}\right)} \\
& \left.+\frac{\left(e^{b K_{e o}}-1\right)\left(K_{21}-K_{e o}\right)\left(K_{31}-K_{e o}\right) e^{-K_{e o} t}}{K_{e o}\left(\lambda_{1}-K_{e o}\right)\left(\lambda_{2}-K_{e o}\right)\left(\lambda_{3}-K_{e o}\right)}\right]
\end{aligned}
$$

Equation (11) is identical to Eq. (2) in the text, which describes the change in amount of drug in the effect compartment with time $\left(d A_{e} / d t\right)$, for the intravenous infusion of a drug that is fitted to a triexponential pharmacokinetic model. For a drug that is fitted to a biexponential pharmacokinetic model, $K_{31}=\lambda_{3}$, so that

$$
\begin{aligned}
\frac{d A_{e}}{d t}= & K_{u e} K_{1 u} K^{0}\left[\frac{\left(e^{b \lambda_{1}}-1\right)\left(K_{21}-\lambda_{1}\right) e^{-\lambda_{1} t}}{\lambda_{1}\left(\lambda_{2}-\lambda_{1}\right)\left(K_{e o}-\lambda_{1}\right)}+\frac{\left(e^{b \lambda_{2}}-1\right)\left(K_{21}-\lambda_{2}\right) e^{-\lambda_{2} t}}{\lambda_{2}\left(\lambda_{1}-\lambda_{2}\right)\left(K_{e o}-\lambda_{2}\right)}\right. \\
& \left.+\frac{\left(e^{\left.b K_{e o}-1\right)\left(K_{21}-K_{e o}\right) e^{-K_{e o} t}}\right.}{K_{e o}\left(\lambda_{1}-K_{e o}\right)\left(\lambda_{2}-K_{e o}\right)}\right]
\end{aligned}
$$

Equation (12) is identical to Eq. (1) in the text.

\section{Nomenclature}

$A_{1}$

$A_{2}$

$A_{3}$

$A_{e}$

$A_{n}$

$A_{u}$

$A_{e s 0}$

$b$

$d A_{e} / d t$

E

$E_{0}$ amount of drug in central compartment amount of drug in peripheral compartment 2 amount of drug in peripheral compartment 3 amount of drug in effect compartment amount of drug in peripheral compartment $n$, where $n$ is equal to an integer greater than 3 amount of drug in urine compartment change in amount of drug in the effect compartment with time producing $50 \%$ of $E_{\max }$ length of time for constant-rate intravenous infusion change in amount of drug in the effect compartment with time effect estimated baseline effect 


$\begin{array}{ll}E_{\mathrm{max}} & \begin{array}{l}\text { maximum effect attributable to the drug } \\ \text { parameter influencing the slope of the dose-effect curve }\end{array} \\ K^{0} & \begin{array}{l}\text { zero-order infusion rate } \\ \text { first-order rate constant for drug transfer from the central to } \\ K_{1 u}\end{array} \\ K_{e o} & \begin{array}{l}\text { frine compartment } \\ \text { First-order rate constant for drug dissipation from the effect }\end{array} \\ K_{i j} & \begin{array}{l}\text { compartment } \\ \text { First-order intercompartmental transfer rate constants, where } \\ i=1,2, \ldots, j=1,2, \ldots, i \neq j\end{array} \\ K_{n r} & \begin{array}{l}\text { First-order rate constant for nonrenal elimination of drug } \\ \text { from the central compartment }\end{array} \\ K_{u e} & \begin{array}{l}\text { First-order rate constant for drug transfer from the urine to } \\ \text { effect compartment } \\ \text { hybrid disposition rate constants such that } \lambda_{1} \text { is the smallest }\end{array} \\ \lambda_{1}, \lambda_{2}, \lambda_{3} & \begin{array}{l}\text { exponent of the } \lambda i \text { 's obtained from the polyexponential } \\ \text { equation } \\ \text { time }\end{array}\end{array}$

\section{REFERENCES}

1. E. Bourke, M. J. A. Asbury, S. O'Sullivan, and P. B. B. Gatenby. The sites of action of bumetanide in man. Eur. J. Pharmacol. 23:283-289 (1973).

2. S. Jayakumar and J. B. Puschett. Study of the sites and mechanisms of action of bumetanide in man. J. Pharmacol. Exp. Ther. 201:251-258 (1977).

3. M. Imai. Effect of bumetanide and furosemide on the thick ascending limb of Henle's loop of rabbits and rats perfused in vitro. Eur. J. Pharmacol. 41:409-416 (1977).

4. S. G. Karlander, R. Henning, and O. Lundvall. Renal effects of bumetanide, a new saluretic agent. Eur. J. Clin. Pharmacol. 6:220-223 (1973).

5. U. B. Olsen. Indomethacin inhibition of bumetanide diuresis in dogs. Acta Pharmacol. Toxicol. 37:65-78 (1975).

6. C. Brater, P. Chennavasin, J. M. Beck, and W. R. Fox. Indomethacin and the response to bumetanide. Clin. Pharmacol. Ther. 27:421-425 (1980).

7. J. Kaufman, R. Hamburger, J. Matheson, and W. Flamenbaum. Bumetanide-induced diuresis and natriuresis: effect of prostaglandin synthetase inhibition. J. Clin. Pharmacol. 21:663-667 (1981).

8. R. Pedrinelli, A. Magagna, F. Arzilli, P. Sassano, and A. Salvetti. Influence of indomethacin on the natriuretic and renin-stimulating effect of bumetanide in essential hypertension. Clin. Pharmacol. Ther. 28:722-731 (1980).

9. D. E. Smith and H. S. H. Lau. Determinants of bumetanide response in the dog: effect of indomethacin. J. Pharm. Sci. 72:1298-1302 (1983).

10. P. A. Friedman and F. Roch-Ramel. Hemodynamic and natriuretic effects of bumetanide and furosemide in the cat. J. Pharmacol. Exp. Ther. 203:82-91 (1977).

11. S. D. Holland and H. E. Williamson. Probenecid inhibition of bumetanide-induced natriuresis in the dog. Proc. Soc. Exp. Biol. Med. 161:299-302 (1979).

12. A. F. Lant. Effects of bumetanide on cation and anion transport. Postgrad. Med. J. Suppi. 51:35-42 (1975).

13. D. C. Brater and P. Chennavasin. Effect of probenecid on response to bumetanide in man. J. Clin. Pharmacol. 21:311-315 (1981). 
14. D. E. Smith and H. S. H. Lau. Determinants of bumetanide response in the dog: effect of probenecid. J. Pharmacokin. Biopharm. 11:31-46 (1983).

15. L. B. Sheiner, D. R. Stanski, S. Vozeh, R. D. Miller, and J. Ham. Simultaneous modeling of pharmacokinetics and pharmacodynamics: application to d-tubocurare. Clin. Pharmacol. Ther. 25:358-371 (1979).

16. B. Whiting, N. H. G. Holford, and L. B. Sheiner. Quantitative analysis of the disopyramide concentration-effect relationship. Br. J. Clin. Pharmacol. 9:67-75 (1980).

17. N. H. G. Holford and L. B. Sheiner. Understanding the dose-effect relationship: clinical application of pharmacokinetic-pharmacodynamic models. Clin. Pharmacokin. 6:429-453 (1981).

18. M. J. D. Powell. A hybrid method for nonlinear equations. In Numerical Methods for Nonlinear Algebraic Equations. P. Rabinowitz (ed.). Gordon and Breach, London, 1970, pp. 90-114.

19. M. J. D. Powell. A Fortran subroutine for solving systems of nonlinear algebraic equations. In Numerical Methods for Nonlinear Algebraic Equations, P. Rabinowitz (ed.), pp. 115-194.

20. C. G. Brodyden. A class of methods for solving nonlinear simultaneous equations. Math. Comp. 19:577-593 (1965).

21. C. M. Metzler, NONLIN, A Computer Program for Parameter Estimation in Nonlinear Situations. Upjohn Co., Kalamazoo, Mich., 1969.

22. J. G. Wagner, Kinetics of pharmacologic response. I: Proposed relationships between response and drug concentration in the intact animal and man. J. Theor. Biol. 20:173-201 (1968). 